\title{
Recomendações de design universal para uso de Mobile User Interface Design Patterns
}

\author{
Leonardo Seiji Nozaki \\ Universidade Federal de São Carlos \\ Sorocaba, São Paulo, Brasil \\ leonardo.nozaki@dcomp.sor.ufscar.br
}

\author{
Luciana Aparecida Martinez Zaina \\ Universidade Federal de São Carlos \\ Sorocaba, São Paulo, Brasil \\ lzaina@ufscar.br
}

\begin{abstract}
RESUMO
O uso de Padrões de Design da Interface do Usuário (User Interface Design Patterns) (UIDP) é visto como uma boa prática para o desenvolvimento de softwares interativos. No entanto, ao aplicar esses padrões, o designer da interface pode introduzir problemas de acessibilidade no software. O objetivo deste trabalho é apresentar o processo de avaliação de um conjunto de recomendações, para uso de UIDP no desenvolvimento de aplicações para dispositivos móveis que evitem a inserção de problemas de acessibilidade.
\end{abstract}

\section{PALAVRAS-CHAVE}

Interação Humano-Computador, User Interface Design Patterns, Acessibilidade, Mobile apps

\section{INTRODUÇÃO}

Acessibilidade é definida como a qualidade ou característica que possibilita às pessoas com deficiência de acessar ou usar algo [4]. Projetar um produto que considere questões de acessibilidade significa observar todos aspectos que permitam que este produto seja usado por pessoas com ou sem deficiências ou limitações situacionais [5]. Limitação situacional é qualquer tipo de problema de acessibilidade que uma pessoa sem deficiência pode experimentar devido a características ambientais como: excesso ou falta de informações na tela, interações através de telas pequenas, entre outras[5]. Dentro do contexto de acessibilidade, o Design Universal determina que a criação de uma solução deve atender às necessidades do maior número possível de situações e públicos. Ao trabalhar com o Design Universal o desenvolvedor de software cria uma única solução buscando atender a todos ou que atenda a maioria dos usuários finais.

Os User Interface Design Patterns (UIDP) tem sido amplamente utilizados no desenvolvimento de aplicações para dispositivos móveis (mobile apps) [6]. Esses padrões são uma coleção de elementos que descrevem soluções para problemas que ocorrem com frequência no design da interface do usuário de mobile apps.

Furtado, Almeida e Marques [3] apontaram dificuldades que usuários possuem em identificar o significado de ícones e as ações relacionadas a eles. No trabalho, os autores concluem que essas

Permission to reproduce or distribute, in whole or in part, material extracted from this work, verbatim, adapted or remixed, as well as the creation or production from the content of such work, is granted without fee for non-commercial use, provided that the original work is properly credited.

IHC 2019 - TRILHA PÔSTERES E DEMONSTRAÇÕES, Outubro 21-25, 2019, Vitória, Brasil. In Anais Estendidos do XVIII Simpósio Brasileiro sobre Fatores Humanos em Sistemas Computacionais. Porto Alegre: SBC.

( 2019 by the author(s), in accordance with the terms of the Creative Commons AttributionNonCommercial 4.0 International Public License (CC BY-NC 4.0). dificuldades podem ser solucionadas com decisões de design mais elaboradas. O trabalho de Silva e Freire [7] tem como foco capacitar designers e desenvolvedores a tornar os mobile apps mais acessíveis para deficientes visuais. Os autores apontam que há falta de técnicas que ajudem os designers e desenvolvedores nesta atividade [7].

No entanto, ao aplicar esses padrões, o designer pode acabar introduzindo problemas de acessibilidade e prejudicar a utilização de mobile apps pelo público geral [2]. Um estudo explorando problemas reportados por desenvolvedores e designers de interface do usuário ao usar UIDP para desenvolvimento de mobile apps, foi reportado em [2]. A partir dos resultados deste estudo, foi elaborado um conjunto de recomendações para uso dos UIDP para desenvolvimento de mobile apps. O foco das recomendações é evitar que ao usar os UIDP os designers introduzam barreiras de acessibilidade em mobile apps. O objetivo deste artigo é apresentar o processo de avaliação do uso desse conjunto de recomendações na visão de designers e desenvolvedores de software. Não é objetivo deste artigo apresentar o processo de criação das recomendações já que este resultado foi reportado em [1].

\section{RECOMENDAÇÕES DE ACESSIBILIDADE E AVALIAÇÃO}

As recomendações emergiram a partir da análise de problemas de acessibilidade reportados por designers/desenvolvedores em um estudo disponível em [2]. As recomendações apresentam uma descrição do UIDP, os problemas de acessibilidade que seu uso pode introduzir e recomendações para que esses problemas sejam evitados. Além disto, imagens e GIFs animados ilustram o problema e a solução para cada recomendação. As recomendações estão disponíveis no site do grupo de pesquisa UXLERIS na forma de catálogo ${ }^{1}$. Um exemplo sucinto desta organização pode ser visto na Tabela 1.

Tabela 1: Exemplo de recomendação para UIDP

\begin{tabular}{|c|c|c|}
\hline UIDP & Problema & Recomendação \\
\hline $\begin{array}{l}\text { Campos de } \\
\text { texto: permitem } \\
\text { que os usuários } \\
\text { digitem e editem } \\
\text { algum texto. }\end{array}$ & $\begin{array}{l}\text { O uso de Placeholder sem rótulo econo- } \\
\text { miza espaço, mas desaparece quando o } \\
\text { usuário começa a digitar, o que remove } \\
\text { instantaneamente todo o contexto do } \\
\text { campo de texto. Eles geralmente são } \\
\text { apresentados com baixo contraste ao } \\
\text { plano de fundo, o que pode estragar a } \\
\text { experiência de usuários com deficiên- } \\
\text { cias visuais ou usuários que estão em } \\
\text { ambientes muito iluminados. }\end{array}$ & $\begin{array}{l}\text { Deve ser feito o uso apro- } \\
\text { priado de rótulo e pla- } \\
\text { ceholder juntos. O rótulo } \\
\text { deve ser curto e descri- } \\
\text { tivo, para o usuário iden- } \\
\text { tificar quais informações } \\
\text { são necessárias. Placehol- } \\
\text { der pode dar mais deta- } \\
\text { lhes sobre o que/como o } \\
\text { usuário deve digitar. }\end{array}$ \\
\hline
\end{tabular}

Para avaliar o uso das recomendações foi realizado um estudo junto a 34 desenvolvedores de software com conhecimento em

${ }^{1}$ http://leris.sor.ufscar.br/accessguide/ 
design de interface. Todos participaram de forma voluntária e assinaram um Termo de Conhecimento Livre e Esclarecido (TCLE) autorizando os pesquisadores a usarem os dados para fins acadêmicos. Para realizar um nivelamento no conhecimento de todos foi realizado um curso de 16 horas divididos em 8 dias. O curso aconteceu em um laboratório de informática da Universidade $\mathrm{Fe}$ deral de São Carlos. Durante o curso foram abordados conceitos teóricos e práticos sobre UIDP, acessibilidade e desenvolvimento de mobile apps. Os participantes aplicaram os conteúdos vistos no curso elaborando protótipos de média fidelidade usando a ferramenta Proto.io ${ }^{2}$. No penúltimo dia do curso as recomendações foram apresentadas aos participantes e eles utilizaram estas para prototipar uma solução de uma mobile apps que contivesse as funcionalidades de reprodução de músicas, playlists, favoritos e outras opções. A Figura 1 apresenta um exemplo produzido por um dos participantes.

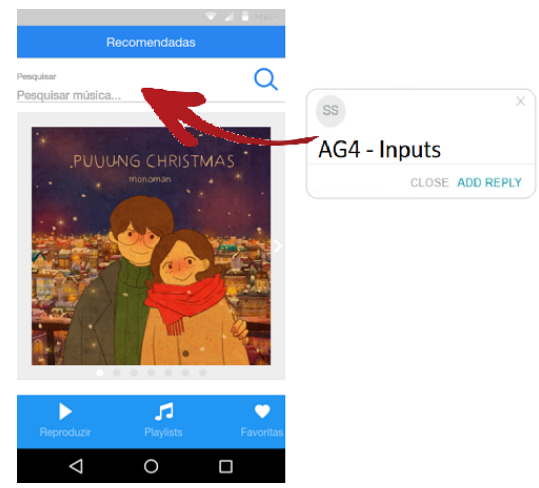

Figura 1: Exemplo de protótipo com comentário

Após prototipação, foi coletado o feedback dos participantes através da técnica Technology Acceptance Model (TAM) [8]. A técnica utiliza um questionário formado por um conjunto de questões dividas em duas dimensões (ver Tabela 2). A primeira foca na utilidade percebida, verificando o grau em que o usuário acredita que usando a tecnologia irá melhorar seu desempenho. A segunda dimensão busca capturar a facilidade de uso percebida, isto é, quão o usuário acredita que usando a tecnologia terá de realizar menos esforço para uma determinada atividade. Para cada questão, o participante deveria atribuir seu grau de concordância que variava entre "Concordo Totalmente" a "Discordo Totalmente".

A Figura 2 apresenta os resultados do questionário TAM. U6 mostrou alta concordância positiva. Foi a que recebeu apenas respostas CT (27 respostas) e CA (7 respostas). A questão U5, recebeu apenas uma resposta CP. Observando os valores das barras de forma geral, nota-se que nenhuma questão recebeu a resposta DT. Além disso, nota-se também que as respostas CT e CA são dominantes. A questão $\mathrm{F} 6$ foi a única que recebeu 2 respostas de discordância. Por fim, a questão F3 foi a que menos recebeu respostas CT (10) e teve alto número de respostas CA (19).

Os resultados mostraram uma boa aceitação por parte dos participantes. Contudo, análises mais detalhadas precisam ser realizadas como trabalho futuro para compreender o porquê em alguns casos

\footnotetext{
${ }^{2}$ https://proto.io/
}

Tabela 2: Perguntas utilizadas no questionário TAM

\begin{tabular}{|c|c|l|}
\hline Dimensão & \multicolumn{2}{|c|}{ Pergunta } \\
\hline \multirow{5}{*}{ Utilidade } & U1 & $\begin{array}{l}\text { Usar as recomendações permitiu que eu criasse mais rapidamente a } \\
\text { solução. }\end{array}$ \\
\cline { 2 - 3 } & U2 & $\begin{array}{l}\text { Usar as recomendações permitiu melhorar minha habilidade quanto à } \\
\text { criação da solução. }\end{array}$ \\
\cline { 2 - 3 } & U3 & $\begin{array}{l}\text { Usar as recomendações melhora minha eficiência quanto à criação da } \\
\text { solução. }\end{array}$ \\
\cline { 2 - 3 } & U4 & Usar as recomendações deixa mais eficaz a criação de solução. \\
\cline { 2 - 3 } & U5 & $\begin{array}{l}\text { Usar as recomendações melhorou minha percepção sobre boas práticas } \\
\text { para a criação da solução. }\end{array}$ \\
\cline { 2 - 3 } & U6 & Considero as recomendações úteis para a criação da solução. \\
\hline \multirow{5}{*}{ Facilidade } & F1 & Foi fácil aprender a utilizar as recomendações. \\
\cline { 2 - 3 } & F2 & Consegui utilizar as recomendações da forma que eu queria. \\
\cline { 2 - 3 } & F3 & As orientações do uso das recomendações são fáceis de entender. \\
\cline { 2 - 3 } & F4 & $\begin{array}{l}\text { Eu entendia o que acontecia durante a minha interação com as reco- } \\
\text { mendações. }\end{array}$ \\
\cline { 2 - 3 } & F5 & Foi fácil ganhar habilidade no uso das recomendações. \\
\cline { 2 - 3 } & F6 & As recomendações permitem flexibilidade para criar os protótipos. \\
\cline { 2 - 3 } & F7 & Considero fácil lembrar as recomendações. \\
\hline
\end{tabular}

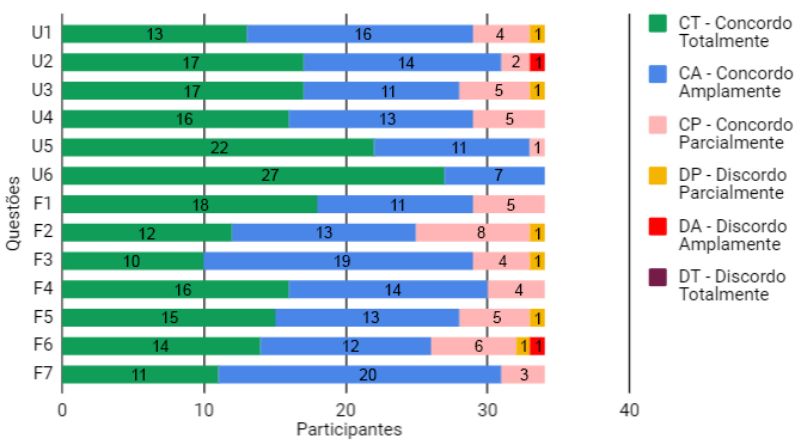

Figura 2: Aceitação do uso da recomendações

os participantes não conseguiram utilizar as recomendações da forma que gostariam.

\section{AGRADECIMENTOS}

Os autores agradecem o suporte financeiro processo $n^{\circ}$ 2018/215590, Fundação de Amparo à Pesquisa do Estado de São Paulo (FAPESP).

\section{REFERÊNCIAS}

[1] Vitor Casadei. 2018. Extending User Interface Design Patterns with accessibility recommendations to guide mobile developers. Dissertação de mestrado, UFSCar, campus Sorocaba (2018).

[2] Vitor Casadei, Toni Granollers, and Luciana A. M. Zaina. 2017. Investigating Accessibility Issues of UI Mobile Design Patterns in Online Communities: A Virtual Ethnographic Study. In Proceedings of the XVI Brazilian Symposium on Human Factors in Computing Systems (IHC 2017). 33:1-33:10.

[3] Alex Furtado, Naila Almeida, and Anna B. Marques. 2018. Investigando a usabilidade de um aplicativo internet banking com a participação de usuários da terceira idade. In Anais Estendidos do XVII Simpósio Brasileiro sobre Fatores Humanos em Sistemas Computacionais.

[4] Shawn L. Henry. 2006. Understanding Web Accessibility. (2006).

[5] Shawn L. Henry, Shadi Abou-Zahra, and Judy Brewer. 2014. The Role of Accessibility in a Universal Web. Proceedings of the 11th Web for All Conference on W4A (2014).

[6] Erik G. Nilsson. 2009. Design patterns for user interface for mobile applications. Advances in Engineering Software 40, 12 (2009), 1318-1328.

[7] Carlos A. Silva and André P. Freire. 2018. Inspeção da Acessibilidade de Aplicativos Móveis Utilizando Software Leitor de Telas. In Anais Estendidos do XVII Simpósio Brasileiro sobre Fatores Humanos em Sistemas Computacionais.

[8] Viswanath Venkatesh and Fred D. Davis. 2000. A Theoretical Extension of the Technology Acceptance Model: Four Longitudinal Field Studies. Management Science 46, 2 (2000), 186-204. 